\title{
The Search for New Drugs from Higher Plants
}

\author{
Kurt Hostettmann* and Andrew Marston
}

\begin{abstract}
Plants have a rich history as medicines for human use. Recently introduced plant-derived drugs include paclitaxel from Taxus (Taxaceae) species for the treatment of cancers, artemisinin for malaria therapy and the alkaloid galanthamine for the management of Alzheimer's disease. A judicious combination of chemical and biological screening techniques can provide a steady supply of lead compounds for the improved treatment of human ailments. By this means, candidate compounds can be rapidly selected for further development, as is the case for xanthones from gentians and coumarins from Peucedanum ostruthium (Apiaceae), which may be of relevance in Alzheimer's disease. Encouraging results are also being obtained for hypericin from Hypericum perforatum $(\mathrm{Hy}-$ pericaceae) in the photodynamic therapy of cancer. An aspect that must not be forgotten is that the usual biological screening procedures do not pick up prodrugs which have to be metabolized by the human body into active analogues. A good example is salicin from Salix species (Salicaceae) which is metabolized in the body to give the anti-inflammatory and analgesic compound salicylic acid. Investigations which include simulation of metabolism are thus of primordial importance.
\end{abstract}

Keywords: Biological screening $\cdot$ Chemical screening $\cdot$ Lead compounds $\cdot$ Metabolism $\cdot$ Plants

\section{Introduction}

Despite the amazing progress in the fields of genomics and genetics, and the importance of synthetic pharmaceutical chemistry and microbial fermentation, plants remain an essential source of new lead compounds. It is estimated that $25 \%$ of prescribed medicines in industrialized countries are of plant origin and some 120 plant-derived compounds from $c a .90$ plant species are used in modern therapy.[1] In fact, statistics show that natural products are implicated in the development of $44 \%$ of all new drugs. Among the $c a$. 300,000 plant species on the earth, only a small percentage has been phytochemically investigated and the frac-

${ }^{*}$ Correspondence: Prof. K. Hostettmann Laboratoire de Pharmacognosie et Phytochimie, Section des Sciences Pharmaceutiques, Ecole de Pharmacie Genève-Lausanne,

Université de Genève,

Quai Ernest-Ansermet 30,

$\mathrm{CH}-1211$ Genève 4

Tel.: +4122 3793401

Fax: +4122379 3399

E-Mail: kurt.hostettmann@pharm.unige.ch tion submitted to biological or pharmacological screening is even smaller. Higher plants are thus a source of thousands of natural products, with an almost infinite variety of different structural variations. ${ }^{[2]}$ These molecules often have specific functions and many of them have biological activities which can be of use to humans. They may provide lead compounds for the development of new drugs or they may be indispensable tools in biomedical research.

\section{New Drugs Derived from Plant Metabolites}

Over the last few decades, the field of anticancer drug research has involved a large amount of work on natural compounds. Thus, in the United States of America, more than $60 \%$ of the approved anticancer drugs between 1983 and 1994 were of natural origin. ${ }^{[3]}$ Among them, several were plant secondary metabolites or molecules derived from plant constituents. The diterpenoid paclitaxel (Fig. 1), previously known as Taxol ${ }^{\circledR}$, was discovered as part of an NCI (National Cancer Institute)sponsored program. It was isolated for the first time from the stem bark of the Pacific yew Taxus brevifolia (Taxaceae) in the late 1960s, but has since been found in other yew species such as the European yew $T$. baccata. Major supply problems have ham- pered, however, the development of this product and approval by the FDA was only obtained in 1992 . While Taxol ${ }^{\circledR}$ was initially used for the treatment of ovarian cancer resistant to chemotherapy, its therapeutic applications now include other gynaecological cancers, as well as lung cancer. Paclitaxel occurs almost exclusively in the stem bark of yews and at very low concentrations. Moreover, a total synthesis is not yet practicable on an industrial scale. The supply problem has been overcome by the development of a semisynthetic approach from 10-deacetylbaccatin II, a more accessible congener found in the needles of $T$. baccata. This strategy has also provided access to structural analogues, one of which, docetaxel (Taxotère ${ }^{\circledR}$ ) has been recently commercialized.

Camptothecin derivatives are the most recent plant-derived substances to be used in cancer therapy (Fig. 1). The monoterpenoid alkaloid camptothecin was isolated in the late 1960s from the Chinese ornamental tree Camptotheca acuminata (Nyssaceae). However, despite potent antitumour properties, the clinical success remained moderate owing to toxic effects and poor solubility. Considerable efforts have since been made to find more active and less toxic structural analogues. Topotecan (Hycamtin ${ }^{\circledR}$ ) was approved in May 1996 by the FDA for the treatment of advanced ovarian cancers that resist other chemotherapy drugs (Fig. 

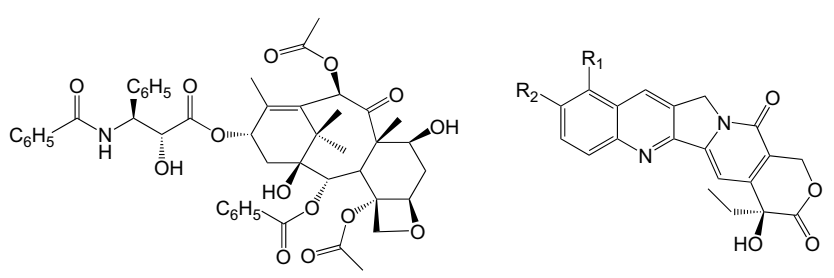

Paclitaxel
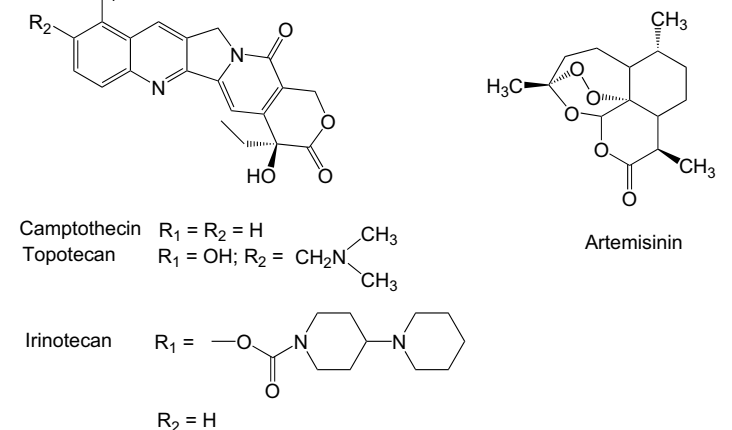

Artemisinin

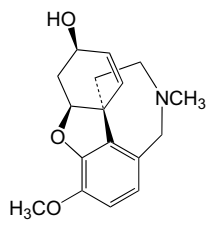

Galanthamine

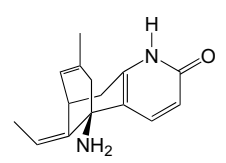

Huperzine A

Fig. 1. Recently introduced drugs of plant origin

1). In June 1996, injectable irinotecan- $\mathrm{HCl}$ was also approved for the treatment of metastatic cancer of the colon or rectum. Further plant-derived agents are currently under investigation for the treatment of cancer. Betulinic acid, a common constituent of birch trees (Betula spp., Betulaceae), for example, has been shown to exhibit potent antitumour activity. This metabolite is considered as a candidate of interest for the treatment of melanoma and other cancers.

In the last decade, plant products have also played a role in the development of new antimalarial agents as illustrated by the discovery and development of artemisinin. ${ }^{[4]}$ Artemisinin, a sesquiterpene lactone containing an endoperoxide group, was isolated in 1972 by Chinese scientists from qinghao (Artemisia annua, Asteraceae), a plant used for over 2000 years in China as a febrifuge and for the treatment of malaria. It represents a completely new chemical class of antimalarial compounds and shows high activity against resistant Plasmodium strains. Because of the highly lipophilic nature of artemisinin, problems were encountered for its administration as a drug. A series of derivatives including ethers and carbonates has been synthesized. Among them, artemether, arteether and sodium artesunate are being licensed as drugs in an increasing number of countries. Artemisinin is also ideal for application with other antimalarials which have a longer span of action, such as the synthetic drug lumefantrine - in so-called Artemisinin Combination Therapy, ACT. The advantage of this approach is that two life cycles of the parasite are covered. One of the most efficient regimens is the artemether-lumefantrine combination (Coartem ${ }^{\circledR}$ ).
Galanthamine (Fig. 1), first isolated in the 1950s from Galanthus nivalis (Amaryllidaceae), [5] is now one of the few therapeutics used in the management of Alzheimer's disease, by a mechanism involving maintenance of acetylcholine levels in the brain. Several randomized, double-blind, parallel group trials comparing galanthamine with placebo have examined the efficacy, the tolerability and the safety of the compound. Galanthamine maintains cognitive function, prevents the onset of newly occurring noncognitive behavioural symptoms for at least one year and delays the loss of activities of daily living by six months. Optimal therapy appears to require early initiation of the drug and a dosage-adjustment period of eight weeks. ${ }^{[6]}$

The clinically interesting alkaloid huperzine A (Fig. 1) was originally isolated from the Chinese clubmoss Huperzia ser- rata (Lycopodiaceae). ${ }^{[7]}$ Huperzine A is a reversible inhibitor of acetylcholinesterase (AChE). The complex between huperzine and the enzyme dissociates rather slowly which could explain its long duration of action. The compound is optically active and in nature only its (-)-enantiomer is present. The synthetic racemic mixture is three times less potent in vitro than the natural compound. A comparable effect is also observed in vivo. However, the racemic compound remains more potent than physostigmine in vitro. It was also shown to protect neurons against amyloid $\beta$-peptide-induced apoptosis. Several clinical trials have been reported in China and the efficacy of huperzine A was demonstrated in the treatment of 447 patients suffering from age-related memory dysfunction or dementia. The most frequently occurring side effects (dizziness, nausea and diarrhoea) were related to its cholinergic properties. No liver and kidney toxicity was detected. ${ }^{[8]} \mathrm{A}$ 5-chloro-vanilline derivative (ZT-1) of huperzine A is currently undergoing clinical trials in Switzerland. This derivative is used in the form of an implant. A multicentre, randomised, double-blind, placebo and active controlled phase II study with subcutaneous injections of the sustained release implants for the treatment of mild to moderate Alzheimer's disease is underway. ${ }^{[9]}$

\section{Approaches for the Discovery of New Drugs from Higher Plants}

In order to find new drugs in plants, it is necessary to screen extracts for the presence of novel compounds and to investigate their biological activities. Once novel compounds are suspected, they are generally isolated in order to have material available for further biological and toxicological testing (Fig. 2).

The path which leads from the intact plant to its pure constituents is long. It involves work which might last anything from

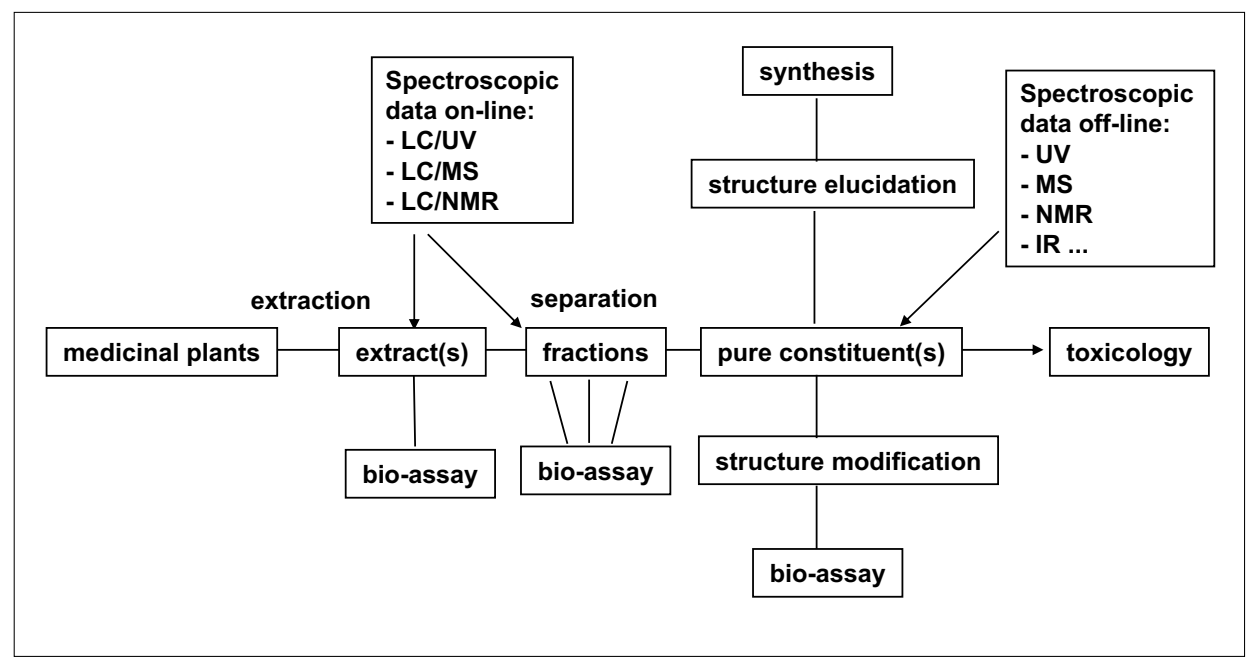

Fig. 2. General scheme for bioactivity-guided isolation 
weeks to years. In order to rationalize and improve the efficiency of the approach, new strategies both in biological screening and chemical screening have to be developed in order to obtain valid chemical and bioactive information prior to the isolation work. ${ }^{[10]}$

\section{Biological Screening with Simple Bioautographic Methods}

In order to identify the active principle(s) of a given plant, access to bioassays that will reveal the mode of action of a given natural product is mandatory. The choice of the correct therapeutic target can, however, be complex since the key enzymes involved in a disease have to be identified. In a phytochemical laboratory it is impossible to have access to all targets and the choice of bioassays has to be restricted to relatively simple and sensitive systems that then bring a rather general idea of the type of activity expected. These types of assays can typically be performed on lower organisms, invertebrates and isolated subcellular systems. ${ }^{[11]}$

At the Laboratory of Pharmacognosy and Phytochemistry (LPP) in Geneva, the array of assays includes the testing for antifungal, antibacterial, molluscidical, insecticidal, larvicidal or antioxidant activities as well as tests for key enzymes such as acetylcholinesterase (AChE).

In order to obtain a rapid localisation of active natural products directly on TLC, which is important to speed up the isolation process, efforts have been made particularly in the development of bioautography methods. ${ }^{[12]}$ With such methods, the crude extracts, fractions or pure constituents are resolved on TLC and the organisms or reagents are applied to the TLC plate. Zones of inhibition can be visualized directly on the TLC, thus revealing the activity. This type of technique has been successfully used for screening antifungal agents inhibiting the growth of a phytopathogenic fungus Cladosporium cucumerinum or the human pathogenic yeast Candida albicans. ${ }^{[12]}$

In the search for new compounds that can be relevant in the pharmacological management of Alzheimer's disease, a bioautographic enzyme assay using AChE has been recently developed. ${ }^{[13]}$ In this test, the enzyme hydrolyses a substrate ( $\alpha$-naphthyl actetate) and the resulting moiety ( $\alpha$-naphthol) is detected as an azo dye. In presence of enzyme inhibitors, no hydrolysis occurs and white spots are observed on the TLC plate. The discovery of bioactivities of extracts and/or fractions by these simple assays is a very useful first step in the process which leads to new pharmacophores. This preliminary data must of course be followed up by more thorough in vitro and in vivo testing but the benchtop bioassays are of great value for guiding the whole pathway from the plant to pure new lead compounds.

\section{Chemical Screening with Hyphen- ated Techniques}

One major drawback of the bioassayguided fractionation strategy is the frequent re-isolation of known metabolites. The chemical screening of crude extracts therefore constitutes an efficient complementary approach allowing localisation and targeted isolation of new types of constituents with potential activities. This procedure, known as dereplication, also enables recognition of known metabolites at the earliest stage of separation, thus avoiding a time-consuming and costly isolation of common constituents.

At the LPP, LC-chemical screening is performed routinely by means of LC/UVDAD, LC/MS and LC/NMR on crude plant extracts (Fig. 3). ${ }^{[14]}$ The dereplication procedure generally proceeds in two steps. The crude plant extracts exhibiting an interesting bioactivity are first analysed by LC/UV/ MS and if complementary structural information is required, de novo structure elucidation can be performed by LC/NMR.

Using such a two-pronged screening approach at the LPP, it has been possible to isolate a certain number of potential lead compounds. In one instance, as part of a programme to find potential therapeuticals from European plants for the management of Alzheimer's disease, several inhibitors of AChE have been isolated from the mountain plant called masterwort, Peucedanum ostruthium (Apiaceae). Fractionation of the crude $\mathrm{CH}_{2} \mathrm{Cl}_{2}$ extract of the roots by centrifugal partition chromatography (CPC) afforded six compounds, final purification of which was by crystallisation or a second CCC step. The pure compounds were then tested against AChE using the bioautographic assay. Solutions of pure compounds

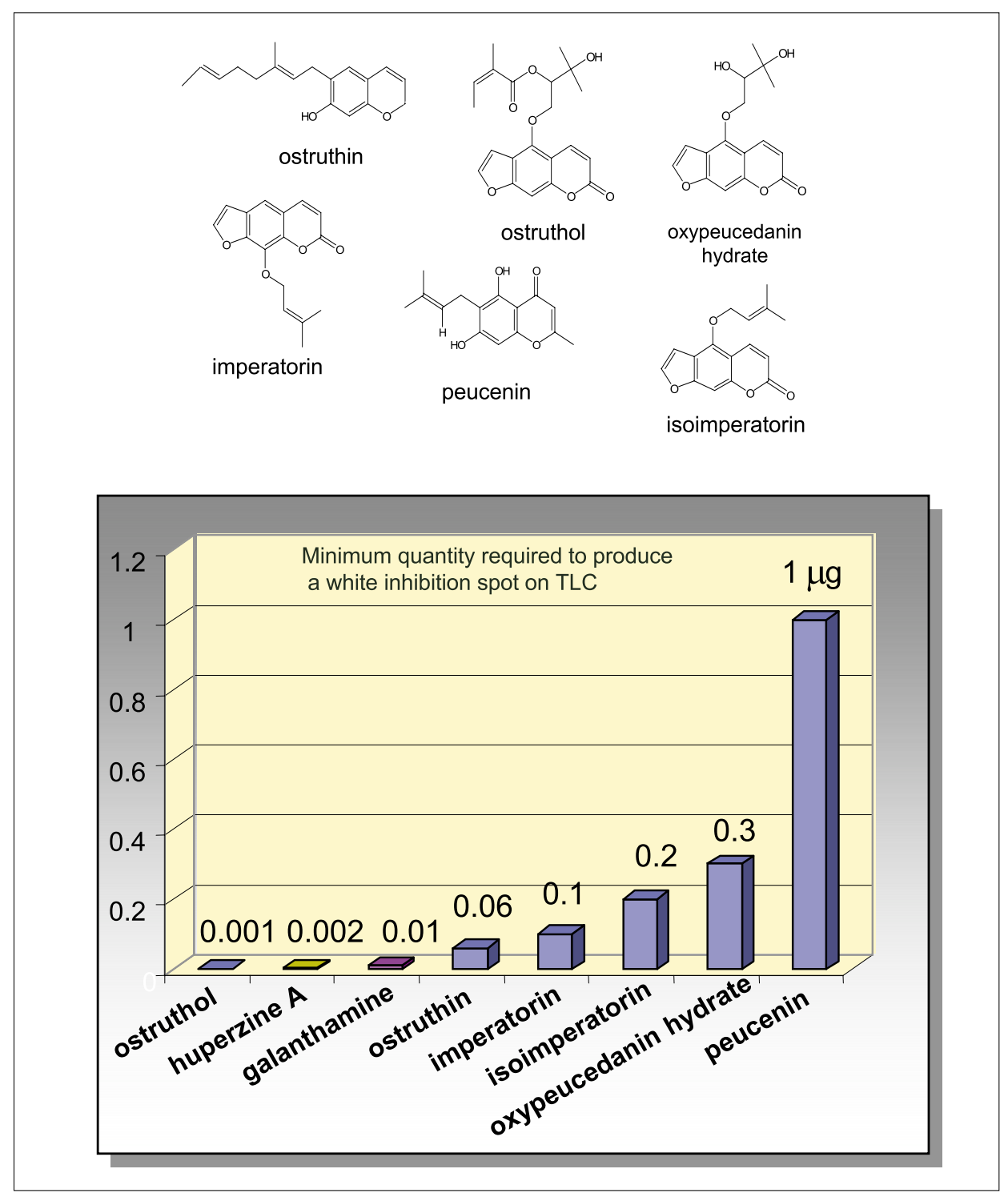

Fig. 3. Inhibition of acetylcholinesterase activity by constituents of Peucedanum ostruthium (Apiaceae) 
were applied on the TLC plate in a quantity of $1 \mu \mathrm{g}$. All the molecules gave an inhibition spot after spraying with enzyme and substrate. Dilutions were then made in order to find the minimum quantity required to produce white inhibition spots. It was shown that the coumarins were more active than the chromone derivative peucenin. Moreover, in this bioassay, ostruthol, the most active of the four tested coumarins, was about ten-fold more active than the AChE inhibitor galanthamine, and almost as strong as huperzine A (from Huperzia serrata, Lycopodiaceae), which is for the moment the most powerful AChE inhibitor.[15]

The methanol extract of Gentiana campestris (Gentianaceae) leaves also exhibited significant inhibition of AChE in the TLC bioassay. A bioactivity-guided fractionation approach was undertaken to isolate the active components, using semi-preparative HPLC. Four xanthones, bellidin, bellidifolin, bellidin-8-O-glucoside (norswertianolin), and bellidifolin-8-O-glucoside (swertianolin) (Fig. 4), were found to be responsible for the anti-AChE effects. A quantity of $1 \mu \mathrm{g}$ of each xanthone deposited on the TLC plate gave an inhibition spot after spraying with enzyme and substrate. Dilutions were then made in order to find the minimum quantity required to produce white inhibition spots. It was found that all xanthones inhibited the enzyme at less than $0.5 \mu \mathrm{g}$. The most active compound, bellidifolin, showed similar activity (inhibition down to $0.01 \mu \mathrm{g}$ ) to galanthamine in this enzyme assay. ${ }^{[16]}$

\section{Killing Cancer by Light}

Certain plants produce photosensitization in animals. When grazing, the animals ingest these plants and begin to suffer from light sickness when exposed to radiation, especially in the 540-610 $\mathrm{nm}$ range. The symptoms include skin efflorescence in the form of blisters and psychomotor excitement.

The phenomenon of photosensitization has been exploited recently in the therapy of two diseases: cancer and age-associated macular degenerescence (AMD).

Photodynamic therapy (PDT) has been described as a promising new modality for the treatment of cancer. PDT involves the combination of a photosensitizing agent (photosensitizer), which is preferentially taken up and retained by tumour cells, and visible light of a wavelength matching the absorption spectrum of the drug. Each of these factors is harmless by itself, but when combined they ultimately produce, in the presence of oxygen, cytotoxic products that cause irreversible cellular damage and tumour destruction. This forms the basis of the therapy with HPD (Visudyne ${ }^{\circledR}$ ) in cancers.

Hypericin, a powerful naturally occurring photosensitizer, is found together with pseudohypericin (Fig. 5) in Hypericum perforatum (Hypericaceae), commonly known as St. John's wort. In recent years increased interest in hypericin as a potential clinical anticancer agent has arisen since several studies have established its powerful in

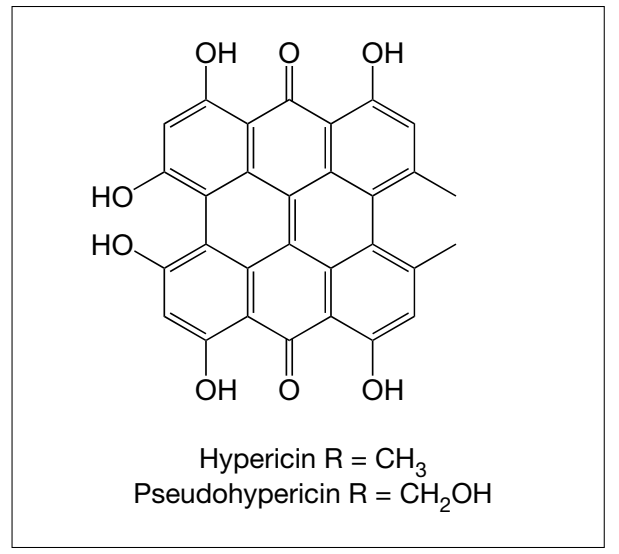

Fig. 5. Structures of hypericin and pseudohypericin vivo and in vitro antineoplastic activity upon irradiation. Investigations of the molecular mechanisms underlying hypericin photocytotoxicity in cancer cells have revealed that this photosensitizer can induce both apoptosis and necrosis in a concentration- and light dose-dependent fashion. Moreover, PDT with hypericin results in the activation of multiple pathways that can either promote or counteract the cell death program. ${ }^{[17]}$

Hypericin has been proposed for the detection and treatment of ovarian and bladder cancer, using a photodynamic therapy approach. Due to its hydrophobicity, systemic administration of hypericin is problematic. Thus, polymeric nanoparticles of polylactic acid or polylactic-co-glycolic acid were used as a drug delivery system.[18]

\section{Prodrugs: An Unexploited Source of Interesting Compounds to $\mathrm{Be}$ Explored}

It is true to say that the anti-inflammatory and analgesic compound salicylic acid (and thus the semi-synthetic derivative aspirin) would not have been discovered in the present style of biological testing and high throughput screening protocols. These rapid techniques are not geared to take into account the metabolism of plant constituents and thus miss the presence of compounds in prodrug form which need activation to produce the required bioactivity. Salicin occurs in the bark of willow (Salix, Salicaceae) species but requires deglucosylation and oxidation in the human body before the active entity, salicylic acid, is formed (Fig. 6).

Another example is the phenolic glucoside arbutin, which is found in the bearberry leaf (Arctostaphylos uva-ursi, Ericaceae). Arbutin is hydrolyzed and oxidized to hydroquinone (Fig. 7) in the human body and then excreted in the form of a glucuronide or a sulphate. The presence of hydroquinone is essential for the treatment of urinary tract infections and in order to have sufficient concentrations $(>60 \mu \mathrm{g} / \mathrm{ml})$, the urine must be alkaline to allow hydrolysis of the conjugates.

Sennosides are glycosides of dianthrones and are found in the dried leaves of Cassia angustifolia and C. senna (Le-

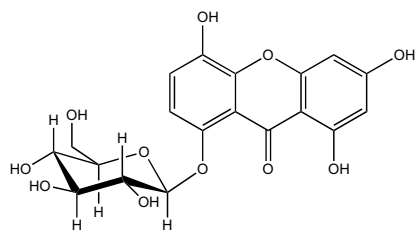

Bellidin-8-O-glucoside
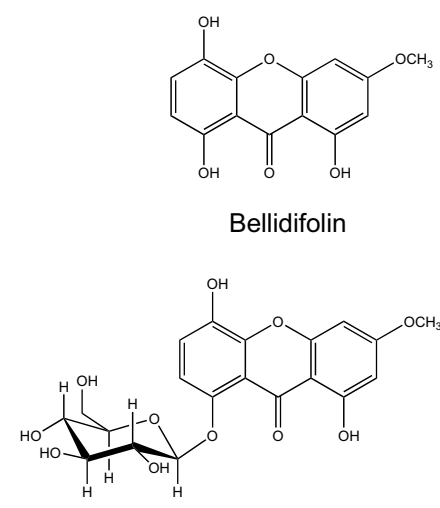

Bellidifolin-8-O-glucoside

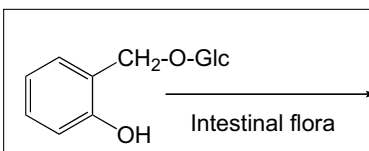

Salicin

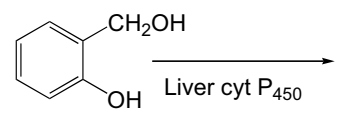

Saligenin

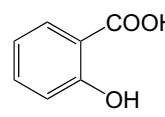

Salicylic acid

Fig. 4. Xanthones with acetylcholinesterase-inhibitory activity from Gentiana campestris (Gentianaceae) 


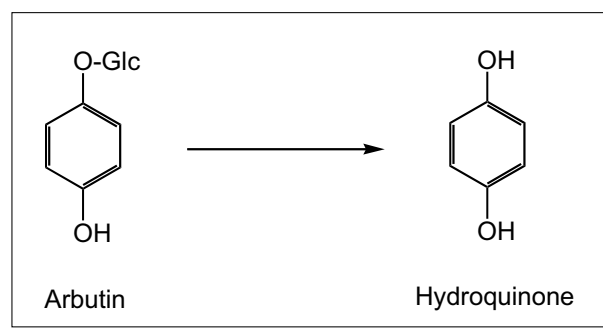

Fig. 7. Conversion of arbutin into the urinary tract disinfectant hydroquinone

guminosae). On ingestion of the herb drug, the inactive sennosides are converted into laxative anthrones by bacteria in the gut. Furthermore, only non-conjugated forms of phytoestrogens (such as isoflavonoids) can exert oestrogen-like activity in animals. Thus, the aglycones have to be liberated by hydrolysis in the stomach or the gut before their effects can be felt. ${ }^{[19]}$

These examples show that in order to exploit Nature's full diversity, an initial activation step is required for many natural products. As well as a classical screening procedure, therefore, the search for new compounds should also include a metabolic simulation with the plant extracts under investigation before studying the bioactivity. This can take the form of a hydrolysis or oxidation step, for instance, and there are many other steps to be looked at.

\section{Conclusion}

Plants still represent a huge reservoir of interesting bioactive lead compounds for the development of therapeuticals. In order to valorise this potential and accelerate the bioactivity-guided isolation process, advanced screening procedures involving both biological and chemical aspects have continually to be developed.

These techniques will also improve the quality and safety control of herbal preparations and precise metabolite profiling of the extracts will become possible. Among the new and exciting challenges which await phytochemists, it is safe to assume that these screening strategies will take an increasingly active part in the discoveries that have yet to be made in natural products chemistry.

\section{Acknowledgements}

Financial support was provided by the Swiss National Science Foundation (Grant no. 200020100083/1 to K. Hostettmann).

Received: March 28, 2007

[1] D. J. Newman, G. M. Cragg, K. M. Snader, Nat. Prod. Rep. 2000, 17, 215.

[2] T. Henkel, R. M. Brunne, H. Müller, F. Reichel, Angew. Chem., Int. Ed. 1999, 38 , 643.

[3] G. M. Cragg, D. J. Newmann, K. M. Snader, J. Nat. Prod. 1997, 60, 52.

[4] K. Borstnik, I. Paik, T. A. Shapiro, G. H. Posner, Int. J. Parasitol. 2002, 32, 1661.

[5] Y.-Z. Shu, J. Nat. Prod. 1998, 61, 1053.

[6] V.Zarotsky, J. J. Sramek, N. R. Cutler, Am. J. Health-Syst. Pharm. 2003, 60, 446.

[7] X. Ma, D.R. Gang, Nat. Prod. Rep. 2004, $21,752$.

[8] X. C. Tang, Y. F. Han, CNS Drug Rev. 1999, 5, 281.

[9] E.Tamchès, J.-M. Orgogozo,D.Wilkinson, S. T. Yancheva, C. Gagiano, P. Grosgurin, H. Porchet, P. Scalfaro, Alzheimer's and Dementia 2006, 2, Supplement 1, S625.

[10] K. Hostettmann, J.-L. Wolfender, C. Terreaux, Pharm. Biol. 2001, 39, 18.

[11] K. Hostettmann, C. Terreaux, Chimia 2000, 54,652 .

[12] L. Rahalison, M. Hamburger, M. Monod, E. Frenk, K. Hostettmann, Planta Med. 1994, 60, 41.

[13] A. Marston, J. Kissling, K. Hostettmann, Phytochem. Anal. 2002, 13, 51.

[14] J.-L. Wolfender, K. Ndjoko, K. Hostettmann, J. Chromatogr. A 2003, 1000, 437.

[15] A. Urbain, A. Marston, K. Hostettmann, Pharm. Biol. 2005, 43, 647.

[16] A. Urbain, A. Marston, E. F. Queiroz, K. Ndjoko, K. Hostettmann, Planta Med. 2004, 70, 1011.

[17] P. Agostinis, A. Vantieghem, W. Merlevede, P. A. M. de Witte, Int. J. Biochem. Cell Biol. 2002, 34, 221.

[18] M. Zeisser-Labouebe, N. Lange, R. Gurny, F. Delie. Int. J. Pharm. 2006, 326, 174.

[19] R. A. Dixon, Ann. Rev. Plant Biol. 2004 $55,225$. 